\title{
Face Recognition Based on Improved LTP
}

\author{
Jiyuan Wang \\ Dept. of Information \& \\ Communication \\ Engineering \\ Tongmyong \\ University \\ Busan, Korea \\ Yuan212108@naver.c
}

om

\author{
Ruyang Zhang \\ Dept. of Information \& \\ Communication \\ Engineering \\ Tongmyong \\ University \\ Busan, Korea \\ dlzry@sina.cn
}

\author{
Tingting Wu \\ Dept. of Information \& \\ Communication \\ Engineering \\ Tongmyong \\ University \\ Busan, Korea \\ Zmt126@hotmail.com
}

\author{
Soo-Yol Ok \\ Dept. of Game \\ Engineering \\ Tongmyong \\ University \\ Busan, Korea \\ Sooyol@tu.ac.kr
}

\author{
Eung-Joo Lee \\ Dept. of Information \\ \& Communication \\ Engineering \\ Tongmyong \\ University \\ Busan, Korea \\ ejlee@tu.ac.kr
}

\begin{abstract}
Localized binary model (LBP) is an efficient local feature description operator. As a nonparametric description operator, it has received more and more attention and has achieved great success in the field of face recognition. In this paper, we introduce only the first-order non-directional feature of LBP operator, and introduce the high-order differential ULDP operator in four directions, and apply the preprocessing method to face recognition. In addition, the threshold for the LBP operator is completely dependent on the defects of the central pixels. In this paper, the local threshold model (ALTP) of the adaptive threshold is proposed. The threshold of the region is automatically generated by calculating the mean and variance of the local region pixels. The experimental results in several commonly used face databases show that ULDP and ALTP in this paper have good robustness to face recognition in nonconstrained environment, especially face recognition with illumination change.
\end{abstract}

\section{Keywords-face recognition; LTP; ALTP; FERET database}

\section{INTRODUCTION}

With the development of modern intelligent information technology, computer and Internet technologies are becoming increasingly developed. In the meantime, people face increasingly strong challenges in information security. In this context, a problem that needs to be solved urgently by the society is about how to identify and affirm identity accurately and fast [1]. At present, personal document or password is the principal method for identity authentication. As is known from long-term practices, the loss of personal documents and passwords has severely threatened the information security of people's daily life and work. In order to find an identity authentication method with higher security, researchers apply biological characteristics to identity authentication through the feature that human characteristics cannot be copied. Subsequently, the method of indentifying biological characteristics enjoys fast development and application. Compared with the traditionally used methods of identifying passwords and personal documents, the biological characteristic recognition has numerous advantages, including being fast, safe, un-duplicated and difficult to be forged [2].

As is known to all, biological characteristics (such as fingerprints, genes, profiles and voices) are characteristics unique to a person. According to such biological characteristics, numerous technical means have been developed and designed based on computer fundamentals in the current phase to identify the human identity, including voice recognition. Currently, the recognition technology in this regard has been widely accepted and applied [3]. In the above-mentioned human characteristic recognition technology, the facial recognition technology receives unanimous acclaims for more friendly interactions and no psychological barrier in use. Secondly, the analysis of human facial expressions helps people to obtain the information that cannot be identified by other methods [4]. In addition, the biological characteristic recognition has several advantages, such as being non-contact, non-mandatory and non-invasive. Moreover, the human face triggers memories more easily than other body parts. Hence the biological characteristic recognition is of great significance to research the facial recognition technology. That's why it is the current hot-spot direction of identity authentication, image processing and other subjects [5].

The exploration of facial recognition technology is of important scientific values, which are represented in two aspects: Firstly, the research on the facial recognition technology deepens people's understandings of humans' visual system; secondly, the research on the facial recognition technology provides a basis for applying artificial intelligence. Hence setting up a facial recognition system on the computer has significant application values and application prospects for identifying facial images [6].

Automatic face recognition (AFR) refers to the use of computer systems to analyze facial images, from which to extract effective authentication information, used to identify the identity of the measured face of a technology [7]. As one of the most direct, the most natural and the most easily accepted biometrics, automatic face recognition has been studied for more than sixty years, especially in the last two decades, as a global pattern recognition and The research focus in the field of computer vision. After several decades of development, automatic face recognition technology has made great progress. The best automatic face recognition system in the ideal situation has been able to achieve satisfactory performance and successfully applied to many security areas. However, a large number of tests and experiments show that: non-constrained 
conditions of face recognition technology there are still serious difficulties. To develop a truly robust and practical automatic face recognition system also needs to solve some of the key issues, in particular, need to study the facial image in a variety of non-ideal environment, the most effective and most resolute feature extraction algorithm.

In this paper, we introduce an Adaptive local ternary pattern (ALTP) for high-performance multi-spectral face recognition. ALTP is also a variant of the local binary pattern feature descriptor. In texture analysis and face recognition, the LBP [8-10] operator can extract features with high resolution. However, in the definition of LBP operator, the threshold in the process of monotonic conversion is the central pixel of the local region, that is, the feature extracted by LBP operator is seriously dependent on the gray value of the central pixel. May result in a region where the gray scale distribution is uniform and the illumination gradient is uniform, and the LBP operator is sensitive to noise. In order to solve this problem, local ternary pattern (LTP) $[11,12]$ proposed to extracting a bifurcation of three values by artificially introducing a threshold to extract richer local resolution feature information. However, the threshold of LTP needs to be set manually, and the threshold is the most important factor affecting LTP operator performance. In face recognition, different images in the face database have different characteristics, it is impossible to use a threshold in the face of all the people has achieved good results. In order to make up for this deficiency of LTP, we proposed ALTP, through the image itself pixel changes, automatically set the threshold, so the use of LTP extracts as much as possible in the resolution of the details of the details

\section{BASIC PRINCIPLES OF LTP}

LBP is an effectively describe operator within the scope of the gray scale of texture, the original operator area is $3 * 3$, the window is mainly composed of a center pixel $g_{c}$ and 8 adjacent pixels $g_{0}, \cdots, g_{7}$. In LBP operator, the texture $T$ of the image can be denoted as [19]:

$$
\mathrm{T} \approx \mathrm{t}\left(\mathrm{g}_{0}-\mathrm{g}_{\mathrm{c}}, \cdots, \mathrm{g}_{7}-\mathrm{g}_{\mathrm{c}}\right)
$$

Will in the window within another 8 pixels to center pixel as a benchmark for banalization operation, concrete process is: the center pixel as the threshold, will the other 8 pixel gray value compared with the threshold, assumptions surrounding pixel size is less than the center pixel, so the pixel tag to 0 , or 1 . After the binary operation, the new $\mathrm{T}$ calculation formula is obtained:

$$
\mathrm{T} \approx \mathrm{t}\left(\mathrm{s}\left(\mathrm{g}_{0}-\mathrm{g}_{\mathrm{c}}\right), \cdots, \mathrm{s}\left(\mathrm{g}_{7}-\mathrm{g}_{\mathrm{c}}\right)\right)
$$

The operation of the binary is expressed as:

$$
\mathrm{S}(\mathrm{x})= \begin{cases}1 & \mathrm{x} \geq 0 \\ 0 & \mathrm{x}<0\end{cases}
$$

Complete formula (3) after operation, eight neighboring pixels in a $3 * 3$ window can be corresponding to the eight binary values, then use the calculation formula (4) weighted summation of pixel location, to calculate the area of LBP values.

$$
\operatorname{LBP}_{\left(\mathrm{x}_{\mathrm{c}}, \mathrm{y}_{\mathrm{c}}\right)}=\sum_{\mathrm{i}=0}^{7} \mathrm{~s}\left(\mathrm{~g}_{\mathrm{i}}-\mathrm{g}_{\mathrm{c}}\right) 2^{\mathrm{i}}
$$

The LBP operator is adopted to calculate the pixels in images and obtain the LBP values that correspond to different pixel positions. In this way, the pattern information of an image is described. The early LBP operator is derived from the locally-nearest concept of pattern. In the early phase, its function is to assist the measurement of the comparison of the local region, namely LBP/C. Fig.4 describes the calculation methods of the early LBP in the early phase in details. The comparative component $\mathrm{C}$ is the mean value of all pixel points in the field that are $\geq$ central pixel points $g_{c}$ and all pixels within the region that are <central pixel points $g_{c}$.

The basic LBP operator has the defect of only covering an extremely small region within the radius. It is thus unable to meet the demands of different sizes and frequency patterns [20]. Ojala et al. conducted the improvement research to make up for the above-mentioned defects, meet the demands for various sizes and meet the requirements of grayness and rotational invariance.

The improvement of local ternary pattern (LTP) is in the quantization stage [13]. By introducing -1 value model, the binary coding of LBP is extended to three-valued coding, and then the image space is transformed in the gray value range to improve the LTP operator extraction. Classification of features [14] LTP artificially introduces a threshold value $t$ to quantize the pixels within the $\mathrm{g}^{\mathrm{c}} \pm \mathrm{t}$ region of the local area center pixel $\mathrm{g}^{\mathrm{c}}$ to 0 ; the pixel whose gray scale is greater than $\mathrm{g}^{\mathrm{c}}+\mathrm{t}$ is quantified to 1 ; the gray value is less than $\mathrm{g}^{\mathrm{c}}-\mathrm{t}$ Of the pixels are quantized to -1 . The three-valued quantization function in LTP can be defined as:

$$
S^{\prime}\left(g, g_{c}, t\right)=\left\{\begin{array}{lc}
1, & g>g_{c}+t \\
0, & \left|g-g_{c}\right| \leq t \\
-1, & g<g_{c}-t
\end{array}\right.
$$

Among them $\mathrm{t}$ is a threshold introduced by man, the introduction of $t$ makes the LTP insensitive to the noise change, but it can be seen from the definition that the LTP gray value of the LTP compared to the monotonic invariance of the LBP gray value transformation Transform and have monotonic invariance [15]. In radius $R$, select $P$ sampling point $\left(x_{c}, y_{c}\right)$ as the center of the local image texture space structure, the specific LTP mode can be calculated using the following formula:

$$
\operatorname{LTP}_{P, R}\left(x_{c}, y_{c}\right)=\sum_{i=0}^{P} 3^{i} \cdot s^{\prime}\left(g, g_{c}, t\right)
$$

By LTP mode definition, LTP coding process and LBP is basically the same, as shown in Fig. 1, the local area of the central pixel value is 85 , we will $\mathrm{T}$ is set to 5 , then the local area of the final ternary The code is: $11011(-1)(-1) 0$ [16]. 


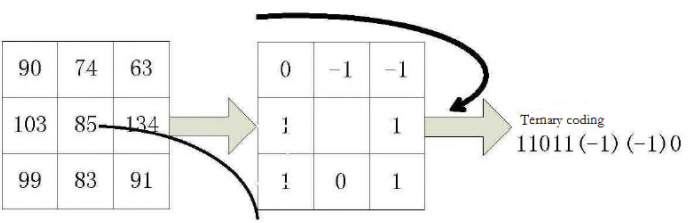

$[85-t, 85+t], t=5$

Fig. 1. LTP operator calculation diagram

According to the definition of LTP mode, it can be seen that the LTP transform of a P-sampling point will eventually produce 3P transform modes, which are very computationally important in image analysis. In order to simplify the calculation process of LTP, the coding of LTP transform can be decomposed according to positive value and a negative value, and the two parts are re-coded with LBP operator.

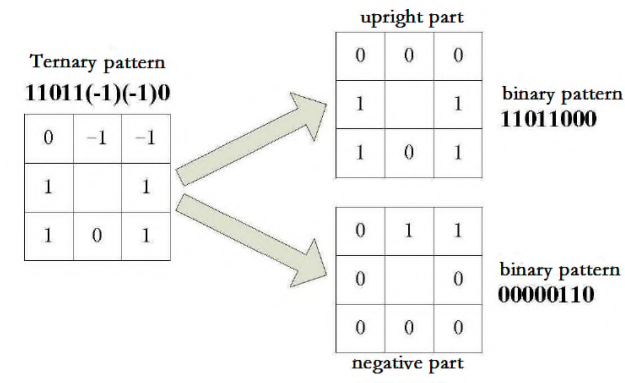

Fig. 2. LTP decomposition process

According to the process shown in the above diagram, it is possible to decompose a LTP mode into two LBP modes. Then, by using the characteristics of LBP such as the unified mode mentioned above, we can decrease the dimension of LTP operator and reduce the LTP Time complexity in analysis. Fig. 2 is the face image LTP transformation diagram, we can see from the figure facial image by LTP transformation and decomposition by LBP can get two images.

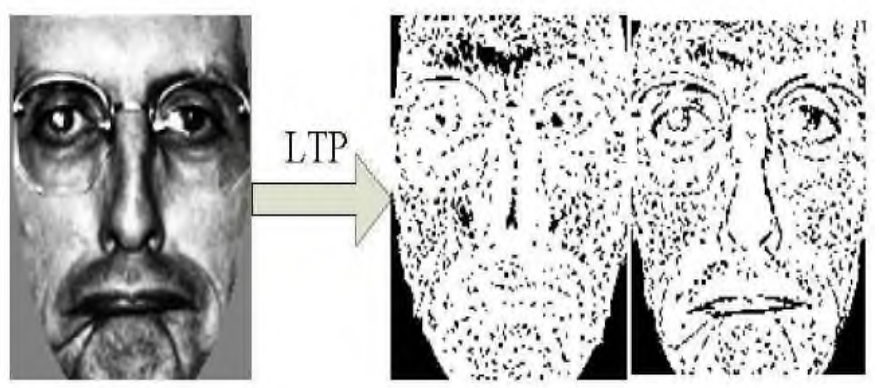

Fig. 3. Example of LTP transformation of face image

\section{ADAPTIVE LOCAL TERNARY PATTERN}

Based on the LBP operator, the LTP operator extends the binary model to the trivalent model by customizing a threshold, which reduces the sensitivity of the operator to the noise to a certain extent. However, the threshold value $t$ needs to be artificially set, and the gray scale distribution of the face image under different conditions is very different. A human-set threshold cannot be applied to the face image under different conditions, LTP in the face recognition application; In addition, the threshold generally needs to go through a large number of experiments to determine, limiting the LTP operator in the actual image analysis applications. In order to solve the above problems, this paper proposes an adaptive local ternary pattern (ALTP).

Different with central thresholds of LBP and LTP, the threshold in ALTP is based on the change in the overall pixel gray value of a local region. That is, the ALTP threshold is different in different local regions. The threshold is mainly determined by the variance of the gray value of the local area pixel and the pixel gray value of the image itself. The triplicate function in ALTP can be defined as:

$$
S_{a}=\left\{\begin{array}{cc}
1 & g_{i}>(\mu+k \sigma) \\
0 & \left|g_{i}\right| \leq(\mu+k \sigma) \\
-1 & g_{i}<(\mu+k \sigma)
\end{array}\right.
$$

And $g_{i}$ is the gray value of the pixels around the central pixel of the local region, $\mu$ is the mean of the local region, $\sigma$ is the standard deviation of the local region, and $\mathrm{K}$ is a constant. In the ALTP, the standard deviation of the pixel gray value of an image as a whole is selected as the value of $\sigma$ in the definition, since it is possible to introduce the change feature of the image as a whole in the ALTP while ALTP extracts the local information feature of the image ALTP extracts some local characteristics while extracting local features, and then extracts more identification information for classification recognition.

ALTP uses the simple statistical method to calculate the threshold of the local area gray value transformation, and the thresholds of different regions are different, so that the image itself can be extracted in the uniform local space of the image and some uniform gradient of the light gradient. In addition, ALTP can automatically calculate the threshold for different images, thus avoiding the artificially set threshold, reduce or eliminate the impact of manual participation in the face recognition process.

Fig. 4 is a process for calculating ALTP, where the mean value of the region is 105.44 , the standard deviation is $65.90, \mathrm{~K}$ is a constant, we set it to 1 , then the corresponding three-valued code is $1100(-1) 000$.

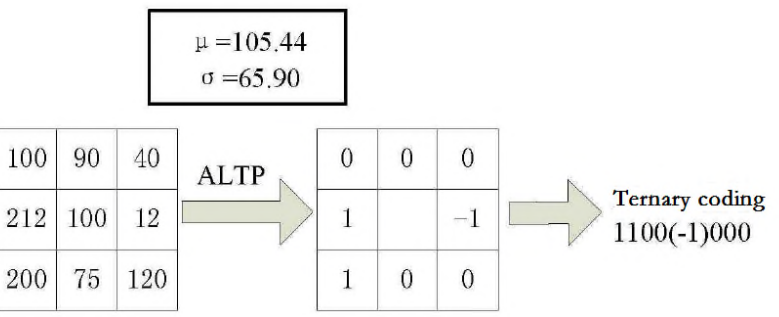

Fig. 4. ALTP calculation process diagram

ALTP only changes the definition of the triple function in the LTP transformation process. A local region is still a series of trilingual coding after ALTP calculations. Compared with LTP, ALTP in the case of $p$ sampling points generated by the number of patterns is still $3 \mathrm{p}$. In order to reduce the number of 
ALTP patterns and simplify the computational complexity of ALTP in face recognition applications, we use the ALTP according to the positive part (defined as: Adaptive Local Ternary Upper Pattern, ALTUP) and The negative part (defined as: Adaptive Local Ternary Lower Pattern, ALTLP) is decomposed, and then the LBP operator is re-encoded for two parts, and an ALTP pattern is decomposed into two LBP modes. Specific decomposition process is shown in Fig. 5:

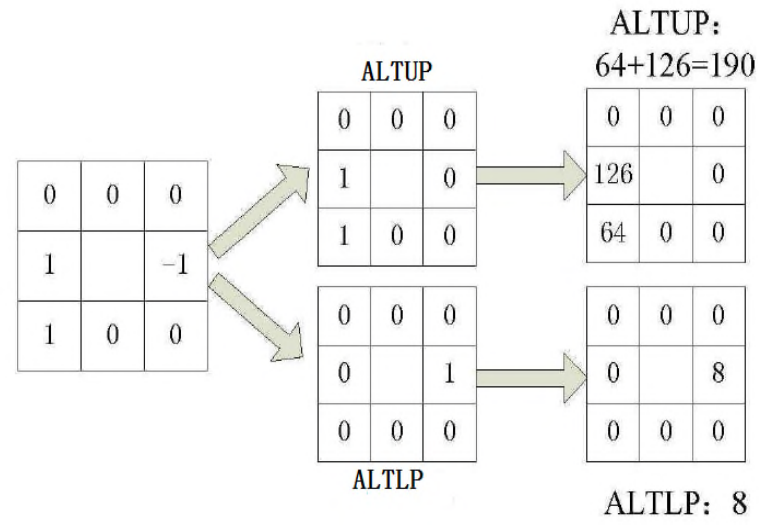

Fig. 5. ALTP decomposition process diagram

Through the decomposition process in the above figure, an ALTP model can be decomposed into two LBP modes. Correspondingly, the concept of unified mode in LBP can be introduced into ALTP-based image analysis, thus limiting the number of patterns generated after ALTP transform, Thus simplifying the computational complexity of ALTP in image analysis. In this paper, ALTP is introduced in face recognition. In face recognition based on ALTP, the face image is divided into several small areas first. Then, the ALTP transform is carried out for each small area and the ALTP mode is obtained after the ALTP transformation is obtained. Then, the corresponding LBP mode is obtained. For image LBP mode of histogram statistics and classification and identification. Fig. 6 is a work of feature extraction based on ALTP in face recognition.

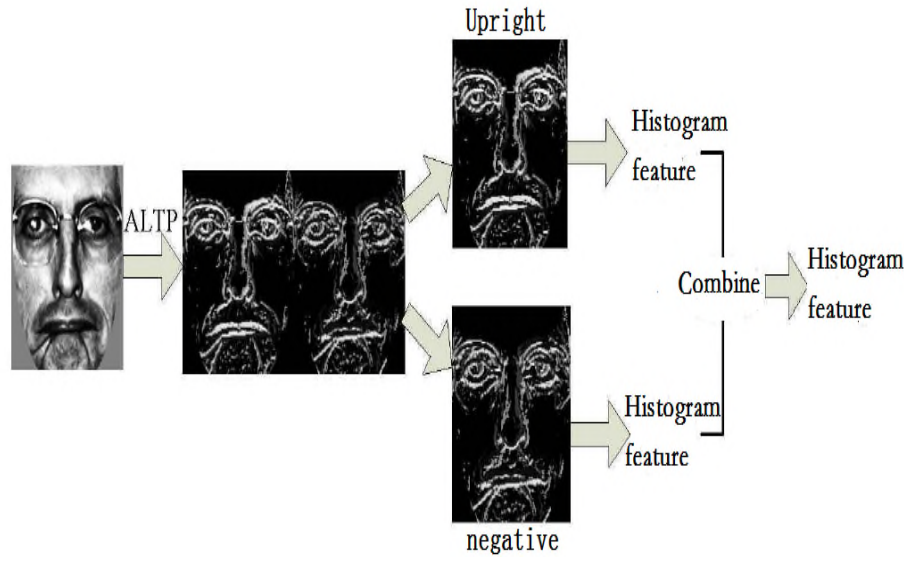

Fig. 6. ALTP extract facial features diagram

\section{EXPERIMENTAL AND ANALYSIS}

In order to verify the performance of the ALTP operator in face recognition in a variety of complex environments, we chose the FERET face database [17, 18]. FERET face database contains 1196 individuals of 14051 gray images. These images are as follows: light changes, facial expressions change, posture changes and age changes. In this experiment, we mainly use the front image in the face database. FERET's frontal image mainly contains the following subsets:

FA subset: contains 1196 people of the front image, mainly used as training images.

FB subset: contains 1195 images, compared with the image in the FA subset, the FB subset of the image contains the facial expression changes.

FC subset: contains 194 images, the image is collected in different lighting conditions.

DUP I subset: contains 722 images, the image is collected in the FA subset in 1 minute to 1031 days after the acquisition.

DUP II subset: contains 234 images, the image is collected in the FA subset in 18 months after the collection.

Select the FA subset of 1196 individuals 1196 positive images composition training set, the other four subsets of all the images constitute a test subset. The parameters in LTP and ALTP are still perform according to the parameters in the previous section.

In the experimental results based on LBP, LDP, LTP and ALTP shown in Fig. 5, the recognition rate of FC-based recognition based on ALTP, the recognition rate of DUP II, and the overall average recognition rate of FERET face database are And the recognition rate of ALTP algorithm is $75.77 \%$, which is $37.11 \%, 17.52 \%$ and $44.84 \%$ higher than that of LBP, LDP and LTP respectively, especially in the FC library (including illumination change). This further illustrates that ALTP operators have better robustness in recognition with illumination changes compared to the other three methods.

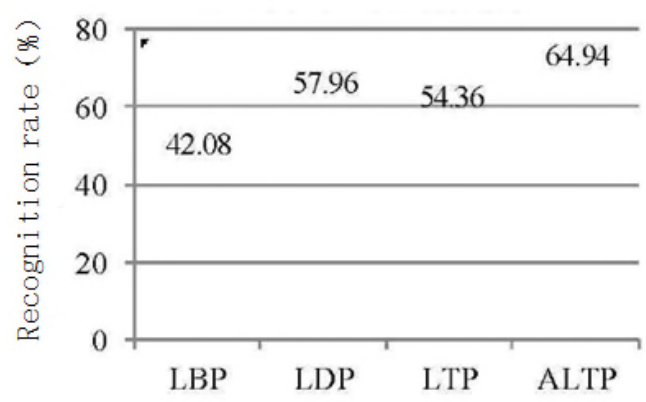

Fig. 7. FERET database average recognition result

\section{CONCLUSION}

This paper presents the basic principle of local trivalent mode LTP and adaptive local trivalent mode ALTP, and introduces ALTP into face recognition. The biggest difference between LTP and LBP operator is that the LTP operator 
introduces the parameters, and the parameter setting needs to be artificially carried out. This will affect the operation of LTP in image analysis. To solve this problem, this paper proposed ALTP operator, the mean of the local area and the standard deviation of the whole image, and automatically calculate the threshold. Experiments show that the ALTP operator has achieved excellent results in face recognition with illumination and expression changes.

\section{ACKNOWLEDGMENT}

This research was supported by the Tongmyong University of Research Grants BB21.

\section{REFERENCES}

[1] A. Fiat and A. Shamir, "How to prove yourself: practical solutions to identification and signature problem," Springer Berlin Heidelberg, pp.186-164, 1986.

[2] R.W. Frischholz and U. Dieckmann, "BioID: a multimodal biometric identification system," Computer, vol. 33, no. 2, pp. 64-68, 2000.

[3] K. Uchida, "Frontiers of image processing technologies and applications. Biometrics personal identification and its application," J. Inst. Electr. Eng. Japan, vol. 120, no. 7, pp. 407-410, 2000

[4] P. Wang and Q. Ji, "Multi-view face and eye detection using discriminant feature," Computer Vision \& Image Understanding, vol. 105, no. 2, pp. 99-111, 2005

[5] P.J. Phillips, P.J. Flynn, T. Scruggs, B.K. Bowyer, J. Chang, K. Hoffman, J. Marques, J. Min and W. Worek, "Overview of the face recognition grand challenge," IEEE Computer Society Conference on Computer Vision and Pattern Recognition, pp. 947-954, 2013.

[6] P.J. Phillips, P. Grother, R. Micheals, D.M. Blackburn, E. Tabassi and M. Bone, "Face Recognition Vendor Test 2002," IEEE International Workshop on Analysis and Modeling of Faces and Gestures, 2003, pp. 44.

[7] R. S. Ghiass, O. Arandjelovic, H. Bendada and X. Maldague, "Infrared face recognition: A literature review," International Joint Conference on Neural Networks, 2013, pp. 1-10

[8] O.T. Pietikainen and M. Harwood, " A comparative study of texture measures with classification based on featured distribution," Pattern Recognition, vol. 29, no. 1, pp. 51-59, Jan 1996.

[9] T. Ojala, M. Pietikäinen and T. Mäenpää, "Multiresolution gray-scale and rotation invariant texture classification with local binary patterns,' European Conference on Computer Vision, 2000, pp. 404-420.

[10] Z. Gou, L. Zhang and D. Zhang, "Rotation invariant texture classification using LBP variance (LBPV) with global matching," Patt. Recogn. vol. 43, no. 3, pp. 706-719, 2010.

[11] B.Z. Zhang, Y.S. Gao. S.Q. Zhao and J.Z. Liu, "Local derivative pattern versus local binary pattern: Face recognition with high order local pattern descriptor," IEEE Trans. Image Proc. vol. 19, no. 2, pp. 533-544, Feb 2010

[12] X. Tan and B. Triggs, "Enhanced local texture feature sets for face recognition under difficult lighting conditions," IEEE Tran. Image Proc. vol. 19, no. 6, pp. 1635-1650, Jun 2010.

[13] M. Yang and L. Zhang, "Gabor feature based sparse representation for face recognition with Gabor occlusion dictionary," European Conference on Computer vision, 2010, pp. 448-461.

[14] Y. Sun, Y. Chen, X. Wang, X. Tang, "Facial Landmark Detection by Deep Multi-task Learning," Springer International Publishing, 2014, pp. 94-108.

[15] M.M. Huang, Z.C. Mu, H. Zeng and S. Huang, "Local image region description using orthogonal symmetric local ternary pattern," Patt. Recogn. Lett. vol. 54, no. 4, pp. 56-62, 2015.

[16] D. Huang, C. F. Shan, M. Ardabilian, Y. H. Wang and L.M. Chen, "Local Binary Patterns and Its Application to Facial Image Analysis: A Survey," IEEE Trans. Syst. Man \& Cybernet. Part C Appl. \& Rev. vol. 41, no. 6, pp. 765-781, 2016.
[17] P.J. Phillips, H. Moon, S.A. Rizvi and P.J. Rauss, "The FERET evaluation methodology for face-recognition algorithms," IEEE Trans. Pattern Anal. Mach. Intell, vol. 22, no. 10, pp. 1090-1104, Oct 2000.

[18] P.J. Phillips, H. Moon, S.A. Rizvi and P.J. Rauss, "The FERET database and evaluation procedure for face recognition algorithms," Imag. Vision Comput. vol. 6, no. 5, pp. 295-306, Apr 1998

[19] X.Y. Tan and B.Triggs, "Enhanced local texture feature sets for face recognition under difficult lighting conditions," IEEE Transactions on Image Processing A Publication of the IEEE Signal Processing Society, vol.19, no.6, (2010), pp.1635-1650.

[20] T. Ahonen, A. Hadid and M. Pietikainen, "Face description with local binary patterns: application to face recognition," IEEE Transactions on Pattern Analysis \& Machine Intelligence, vol.28, no.12, (2006), pp.2037-2041. 